\title{
Incentive mechanism design for citizen reporting application using Stackelberg game
}

\author{
I Made Ariya Sanjaya ${ }^{1}$, Suhono Harso Supangkat ${ }^{1}$, Jaka Sembiring ${ }^{2}$, Widya Liana Aji ${ }^{3}$ \\ ${ }^{1}$ School of Electrical Engineering and Informatics, Institut Teknologi Bandung, Bandung, Indonesia \\ ${ }^{2}$ Center of City Innovation and Smart Community (PIKKC) Institut Teknologi Bandung, Bandung, Indonesia \\ ${ }^{3}$ PT. Kazee Digital Indonesia, Bandung, Indonesia
}

\begin{tabular}{l}
\hline Article Info \\
\hline Article history: \\
Received Feb 28, 2021 \\
Revised Jul 22, 2021 \\
Accepted Aug 9, 2021 \\
\hline Keywords: \\
Citizen reporting \\
City issue \\
Incentive mechanism \\
Mobile crowdsensing \\
Stackelberg game
\end{tabular}

Article Info

Received Feb 28, 2021

Revised Jul 22, 2021

Accepted Aug 9, 2021

City issue

Mobile crowdsensing

Stackelberg game

\begin{abstract}
The growing utilization of smartphones equipped with various sensors to collect and analyze information around us highlights a paradigm called mobile crowdsensing. To motivate citizens' participation in crowdsensing and compensate them for their resources, it is necessary to incentivize the participants for their sensing service. There are several studies that used the Stackelberg game to model the incentive mechanism, however, those studies did not include a budget constraint for limited budget case. Another challenge is to optimize crowdsourcer (government) profit in conducting crowdsensing under the limited budget then allocates the budget to several regional working units that are responsible for the specific city problems. We propose an incentive mechanism for mobile crowdsensing based on several identified incentive parameters using the Stackelberg game model and applied the multi-objective optimization problem (MOOP) to the incentive model in which the participant reputation is taken into account. The evaluation of the proposed incentive model is performed through simulations. The simulation indicated that the result appropriately corresponds to the theoretical properties of the model.
\end{abstract}

This is an open access article under the $C C B Y$-SA license.

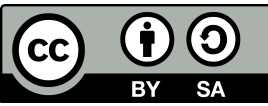

\section{Corresponding Author:}

I Made Ariya Sanjaya

School of Electrical Engineering and Informatics, Institut Teknologi Bandung

Bandung, 40132, Indonesia

Email: byz_ariya@students.itb.ac.id

\section{INTRODUCTION}

The increase in the urban population in Indonesia increases the complexity of city problems. As a developing country, cities in Indonesia face a vast amount of problems, from small-scale and simple issues such as trash collection to large-scale and complex problems such as economic development. To solve the various city issues effectively, the cooperation between citizens and the government is required [1]. The utilization of smartphones in terms of mobile crowdsensing enables such a cooperation where the citizens provide information regarding the city issues, while the government collects and analyzes information to gain insights of the various city issues which could then be used for city planning as well as its policies. Figure 1 shows the basic crowdsensing system that consists of a crowdsourcer referred to the government and the participants using their smartphones to perform the sensing service. Both of them are connected via the cloud.

To motivate citizens' participation in crowdsensing and compensate them for their resources, such as battery consumption and data transmission costs, it is necessary to incentivize the participants for their sensing service. Previous studies have contributed to the development of the incentive mechanism on mobile crowd- 
sensing, such as the proposed model on the resource-aware incentive mechanism [2], two credit-based privacyaware incentive schemes [3], and the simple endorsement web to incentivize the participants [4]. Nevertheless, the challenges to incentivize the crowdsensing participant remain open as discussed in [5].

In addition to the challenges to incentivize the participant, another challenge is to optimize crowdsourcer (government) profit in conducting crowdsensing under the limited budget then allocates the budget to several regional working units that are responsible for the specific city problems. For instance, the environmental working unit is responsible for the environmental problems in the city. To address this issue, we propose the multi-objective optimization problem (MOOP) [6] to design an incentive mechanism for mobile crowdsensing regarding the city issues.

To design an incentive model, one can model the interaction between participants and a crowdsourcer as a Stackelberg game model. Under this model, the crowdsourcer as the leader moves first and decides its strategy; then, the participants as followers pick their best response strategy according to the leader strategy, whereas the objective of both the leader and followers is to maximize their utility. There are several studies that used the Stackelberg game to model the incentive mechanism [4], [7]-[13]; however, those studies did not include a budget constraint for limited budget case.

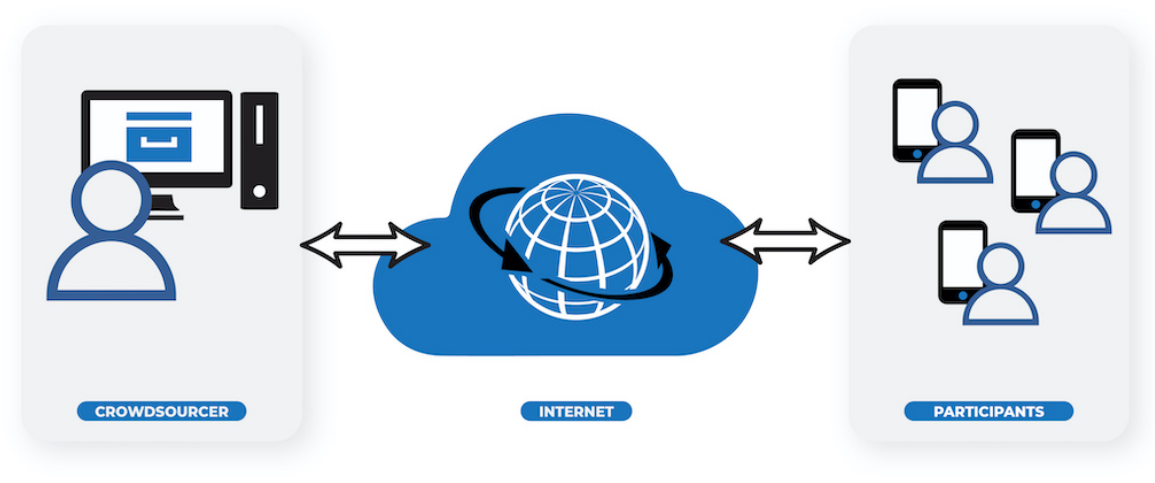

Figure 1. Mobile crowdsensing system

\section{RELATED WORKS}

There are many studies concerning incentive mechanism design for mobile crowdsensing [14] from multi-armed bandit to game theoretical approaches. Gao et al. [15] studied an unknown worker recruitment process as a combinatorial multi-armed bandit problem to determine the recruiting strategy that maximizes the weighted sum of the completion qualities under a limited budget. Wu et al. [16] proposed a context-aware multi-armed bandit incentive mechanism to facilitate quality-based worker selection. Auction games to design incentive mechanisms are also widely used. Jin et al. [17] designed an incentive mechanism based on the single-minded reverse combinatorial auction to preserve the privacy of the worker's bid against the other honestbut-curious workers. Jin et al. [17] proposed the user-centric model auction-based incentive mechanism which is computationally efficient, individually rational, profitable, and truthful. Liu et al. [18] proposed IMRAL, i.e., an incentive mechanism based on reverse auction for location-aware sensing, to enhance the willingness of users to participate in crowdsensing by maximizing their expected profits. Another game theoretical approach used in constructing incentive mechanisms is the Stackelberg game [4], [7]-[13].

Previous work by [7] provided an efficient algorithm to compute the unique Stackelberg equilibrium at which the crowdsourcer utility is maximized. The extension of this work is provided by [8], [19] to include privacy considerations and spatial coverage of the collected dataset. However, the optimization of the crowdsourcer utility in these previous works is less relevant for the case when we want to optimize the profit of several regional working units simultaneously. In this case, the notion of multiple crowdsourcers is more appropriate. A study by [20] provided an incentive mechanism with multiple crowdsourcers but did not include the budget constraint. In addition, all of these studies did not consider the participant reputation.

Our work on the incentive model is related to the model provided by [7], [8], [19], [20]. We extend the existing models by taking the multiple crowdsourcers into account, where each of those represents a certain 
issue category and thus relates to the regional working unit, and by imposing a budget constraint on the model since it is more relevant in real situations. The multiple crowdsourcers case with the budget constraints allows us to apply multi-objective optimization [21], [22] by considering multiple crowdsourcer objective functions so that we can optimize the profit or utility of each regional working unit simultaneously under a given budget.

\section{SYSTEM MODEL AND PROBLEM FORMULATION}

In this study, we designed an incentive mechanism as follows:

- It classifies incentives according to issue categories.

- It considers the participant's reputation and encourages the participant to report the prioritized issues at a certain period.

- For a given allocated budget, it maximizes the utility of both the participant and the government in each category of the issue which corresponds to the regional working unit.

\subsection{System model}

The crowdsensing system consists of $N$ participants and a single crowdsourcer (government) who categorized issues into $M$ categories. For instance, there are 17 issue categories as mention in [23] Let $P=\{1,2, \cdots, N\}$ be the set of participants and $C=\left\{c_{1}, c_{2}, \cdots, c_{M}\right\}$ be the set of issue categories where $N, M \geq 2$. In this study, we apply a crowdsourcer-centric model [7], [8], [19], [20]. We use Figure 2 to represent the system.

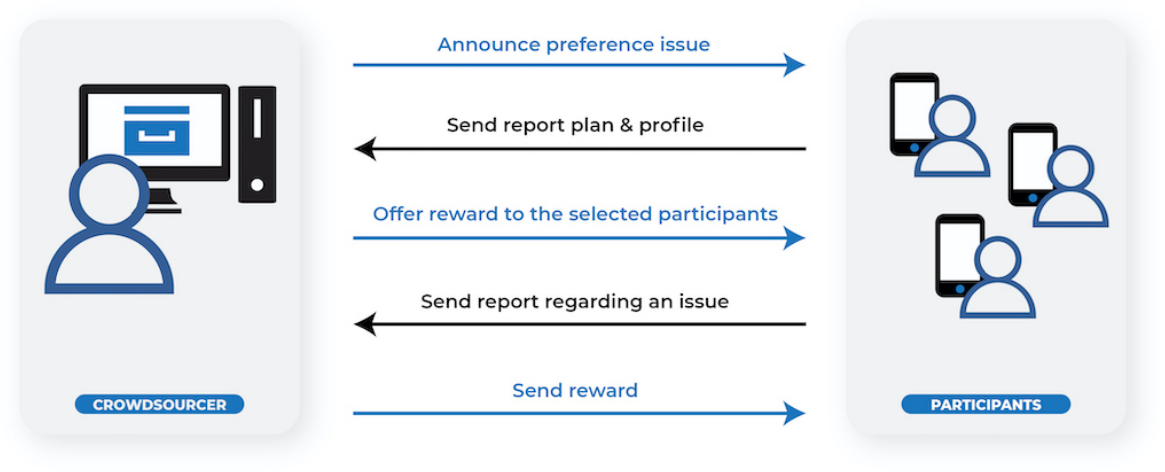

Figure 2. Interaction between participant and crowdsourcer in the incentive model

The crowdsourcer first announces $m$ prioritized (preference) issues $c_{p} \in C, m \geq 1$. The participant $i$ subsequently makes its report plan consisting of the category of issue $c_{i} \in C$ that he/she wants to participate in and the associated cost per bit of data $\kappa_{i} \in\left(0, \kappa_{\max }\right]$ and then submits it to the crowdsourcer. In this case, the participant is free to choose whether he/she wants to report the issue in the prioritized category or another issue that he/she encounters. For instance, if the participant wants to report the broken bridge in his/her town, he/she can select the infrastructure issue in the set of issue categories. We assume that participants are rational and noncooperative, i.e., each participant is only interested in maximizing its utility and if there is not a sufficient reward for reporting an issue, he/she will not contribute in crowdsensing. For each participant, the crowdsourcer knows its profile in terms of its reputation, which is elaborated later. The collection of data (issue category, cost, and reputation) provided by each participant enables the system to compute the optimal set of participants that maximizes the utility of both the participant and the crowdsourcer. The selected participant from the optimal set is then offered some reward for some amount of quality of report $q_{i}$ that he/she is expected to submit; otherwise, it would affect his/her reputation. After submitting the data, the selected participant receives the payment. In this case, the report quality is determined by the size of the content type of the report, such as the breadth of description, in which we might analyze the details of the text description, and the image resolution or video frame rate and its resolution, in which we might analyze the field of view from an event in a report [24]. 
We apply the Stackelberg game to model the incentive mechanism. In this case, the crowdsourcer is a leader and the participants are followers. The crowdsourcer and participants are players of the game. Each player has its own strategy. The crowdsourcer's strategy is the reward for each category of issues, denoted by $\mathbf{R}=\left(R_{c_{1}}, R_{c_{2}}, \cdots, R_{c_{M}}\right)$, whereas the strategy of the participant $i$ is the quality of report $q_{i} \geq 0$ of an issue category that he/she has an interest in participating. The crowdsourcer moves first and decides its strategy in terms of reward $\mathbf{R}$. The participants then pick their best response strategy of the quality of the report on a certain issue to maximize their own utility.

We assume that the reward received by participant $i$ is proportional to $q_{i}$, its reputation $\gamma_{i}$, and the parameter associated with the prioritized issue for participant $i$, denoted by $\alpha_{i}$. Let $V_{p}$ be the set of participants that report the issue on the prioritized category and $\alpha \in(0,1)$; then, $\alpha_{i}$ is defined by

$$
\alpha_{i}= \begin{cases}\alpha, & \text { if } i \in V_{p} \\ 0, & \text { otherwise }\end{cases}
$$

which provides more reward for the participant who reports the issue on the prioritized category to motivate sending the report on the prioritized issue. The reputation of participant $i$, denoted by $\gamma_{i}$, is defined by

$$
\gamma_{i}= \begin{cases}1, & \text { for new participant } \\ \frac{\text { number of approved reports }}{\text { number of selected report plans }}, & \text { otherwise. }\end{cases}
$$

in which $\gamma_{i} \in\left[\gamma_{\min }, 1\right]$ and $\gamma_{\min }$ is the minimum reputation required for a participant to contribute in the crowdsensing. We define a new participant as the participant who is just active in crowdsensing for the first $p$ times. Suppose $p=4$; then, the reputation of the participant is 1 when he/she contributes to crowdsensing in the first four times. In this case, the reputation of the new participant is assigned to the maximum value to provide the opportunity for a new participant to contribute in the crowdsensing. We assume that in the first $p$ times, there is at least one report plan from the new participant to be selected. For other participants, $\gamma_{i}$ is proportional to the number of approved reports. The approved report is the report that provides a satisfactory representation of an event/issue from the analysis of its content. The participant whose report plan is selected but does not send the report or its report has a poor representation of the event/issue will gain a lower reputation than the participant whose report is approved.

We refer the utility function of the participant to [7], [8] which assume that the reward is proportional to $q_{i}$ and the cost function is linear for participant utility. The utility function (i.e., reward minus cost) of the participant $i$ interested in reporting an issue on category $c_{j}$ is given by the following (3)

$$
u_{i}=\frac{\left(1+\alpha_{i}\right) \gamma_{i} q_{i}}{\sum_{k \in V_{j}} \gamma_{k} q_{k}} R_{c_{j}}-\kappa_{i} q_{i}
$$

where $R_{c_{j}}$ is the reward of issue category $c_{j}$ and $V_{j}$ is the set of participants that report an issue on category $c_{j}$. The utility function of the crowdsourcer for each category of the issue is

$$
u_{c_{j}}=\lambda \ln \left(1+\sum_{i \in V_{j}} q_{i}^{y}\right)-R_{c_{j}}
$$

with the constraints

$$
\sum_{c_{j} \in C}\left(1+\alpha_{c_{j}}\right) R_{c_{j}} \leq R_{\text {budget }}
$$

and

$$
R_{c_{j}} \geq 0 \text { for } c_{j} \in C
$$

where $\lambda>1$ is a system parameter, $y \in(0,1)$ is the rate of the crowdsourcer's diminishing return on the participant's quality of the report, whereas the ln term reflects the crowdsourcer's diminishing return on the number of participants. $\alpha_{c_{j}}=\alpha$ for the prioritized category; otherwise, it is zero. $R_{b u d g e t}$ is the government allocated budget to conduct the crowdsensing. Constraint (5) tells us that the total reward of each issue category is at most as much as $R_{\text {budget }}$ in which the term $\left(1+\alpha_{c_{j}}\right)$ provides the additional allocation reward for the prioritized issues. It is obvious that $R_{c_{j}}$ is nonnegative, as given by constraint (6). 


\subsection{Optimization problem formulation}

The objective of the crowdsourcer is to maximize its utility for each category of the issue for a given allocated budget by determining the optimal reward $\mathbf{R}=\left(R_{c_{1}}, R_{c_{2}}, \cdots, R_{c_{M}}\right)$, whereas the participant assigns the amount of $q_{i}$ to maximize its utility after committing to report an issue on category $c_{j}$. The Stackelberg game optimization problem can be written as follows:

- Let $\lambda, y, \alpha_{c_{j}}$, and $R_{b u d g e t}$ be given. For each category of issue, the crowdsourcer aims to

$$
\begin{array}{ll}
\underset{\mathbf{R}}{\operatorname{maximize}} & u_{c_{j}}=\lambda \ln \left(1+\sum_{i \in V_{j}} q_{i}^{y}\right)-R_{c_{j}}, \forall c_{j} \in C, \\
\text { subject to } & \sum_{c_{j} \in C}\left(1+\alpha_{c_{j}}\right) R_{c_{j}} \leq R_{\text {budget }}, R_{c_{j}} \geq 0 .
\end{array}
$$

- Let $\alpha_{i}, \kappa_{i}, R_{c_{j}}, \gamma_{i}, \gamma_{-i}$, and $q_{-i}$ be given. $\gamma_{-i}$ and $q_{-i}$ are the set of reputation and quality reports other than that of participant $i$, respectively. For a certain issue category $c_{j}$, the participant aims to

$$
\begin{array}{ll}
\underset{q_{i}}{\operatorname{maximize}} & u_{i}=\frac{\left(1+\alpha_{i}\right) \gamma_{i} q_{i}}{\sum_{k \in V_{j}} \gamma_{k} q_{k}} R_{c_{j}}-\kappa_{i} q_{i}, \\
\text { subject to } & q_{i} \geq 0
\end{array}
$$

\section{PROBLEM RESOLUTION}

The objective of both the crowdsourcer and the participant leads to the notion of the Stackelberg equilibrium, i.e., the equilibrium such that no player can increase its utility by unilaterally changing its strategy which is associated with the pair of $\mathbf{R}$ and $\mathbf{q}=\left(q_{1}, q_{2}, \cdots, q_{n}\right)$ that maximizes the utility of both the crowdsourcer and the participant for a given category of issue. We solve the Stackelberg game problem in the previous section by using backward induction where the crowdsourcer considers all possible best responses of the participants (if they exist), i.e., the strategy that maximizes each of the participant's utility on a certain issue category regardless of the strategy of other participants; then, the leader chooses its strategy that maximizes its utility according to that knowledge. The best response of the participant after choosing a certain issue category is its strategy at Nash equilibrium, which is defined as follows:

Definition 1 For an issue category, a set of strategies $\mathbf{q}^{n e}=\left(q_{1}^{n e}, q_{2}^{n e}, \cdots, q_{n}^{n e}\right)$ is a Nash Equilibrium (NE) if for any participant $i$ in that category

$$
u_{i}\left(q_{i}^{n e} ; q_{-i}^{n e}\right) \geq u_{i}\left(q_{i} ; q_{-i}^{n e}\right)
$$

for any $q_{i} \geq 0$.

$q_{-i}^{n e}$ denotes the set of quality of reports at NE other than $q_{i}^{n e}$. In fact, under this model, the strategy at Nash equilibrium of a participant $i$ that commits to report an issue on category $c_{j}$ exists and is unique [7], [8] which is given by (9):

$$
q_{i}^{n e}= \begin{cases}0, & \text { if } i \notin S_{j} \\ \frac{\left(\left|S_{j}\right|-1\right)\left(1+\alpha_{i}\right) R_{c_{j}}}{\gamma_{i} \sum_{m \in S_{j}} \frac{\kappa_{m}}{\gamma_{m}}}\left(1-\frac{\left(\left|S_{j}\right|-1\right) \frac{\kappa_{i}}{\gamma_{i}}}{\sum_{m \in S_{j}} \frac{\kappa_{m}}{\gamma_{m}}}\right), & \text { otherwise }\end{cases}
$$

provided by (10),

$$
\frac{\kappa_{i}}{\gamma_{i}}<\frac{\sum_{m \in S_{j}} \frac{\kappa_{m}}{\gamma_{m}}}{\left|S_{j}\right|-1}, \text { for } i \in S_{j}
$$

where $S_{j}$ is the set of participants such that its report plan is selected on issue category $c_{j}$ and $\left|S_{j}\right| \geq 2$.

The algorithm to compute the strategy of the participant at Nash equilibrium is provided in [7], [8]. Since we differentiate the participants based on the issue category he/she has chosen, i.e., whether it is a prioritized issue or not characterized by a factor $\alpha$, we take the parameter prioritized category $\alpha_{i}$ into account in the calculation of the report quality at NE given by algorithm 1. By applying (9) in the calculation, the report quality at $\mathrm{NE}$ of the participants choosing the prioritized issue differs by a factor $(1+\alpha)$ from those who choose the nonprioritized issue category. The crowdsourcer expects a higher report quality for participants who are interested in reporting the issue in the prioritized category than those who report the nonprioritized issue. If 
there is a case in which only one participant sends the report plan to a certain issue category, the crowdsourcer might set an arbitrary value of $q_{i}$, for instance, $q_{i}=1$; then, the participant's utility is $\left(1+\alpha_{i}\right) R_{c_{j}}-\kappa_{i} \approx$ $\left(1+\alpha_{i}\right) R_{c_{j}}$ given by $\kappa_{i} \ll\left(1+\alpha_{i}\right) R_{c_{j}}[20]$.

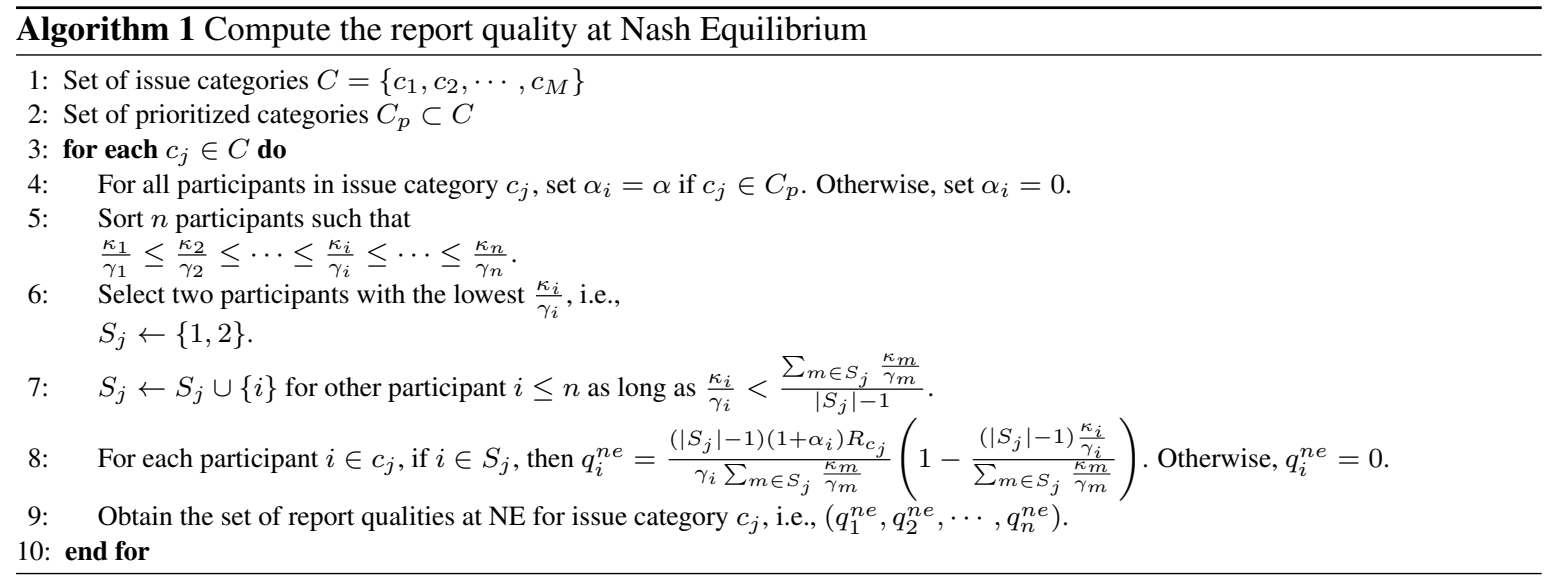

From (9), we know that there is a unique strategy for each participant to maximize its utility. Therefore, the crowdsourcer can choose the optimal strategy according to his knowledge about each participant's best response. We might rewrite (4) as (11),

$$
u_{c_{j}}=\lambda \ln \left(1+R_{c_{j}}^{y} \sum_{i \in S_{j}} T_{i}^{y}\right)-R_{c_{j}} \text { for } c_{j} \in C,
$$

where

$$
T_{i}=\frac{\left(\left|S_{j}\right|-1\right)\left(1+\alpha_{i}\right)}{\gamma_{i} \sum_{m \in S_{j}} \frac{\kappa_{m}}{\gamma_{m}}}\left(1-\frac{\left(\left|S_{j}\right|-1\right) \frac{\kappa_{i}}{\gamma_{i}}}{\sum_{m \in S_{j}} \frac{\kappa_{m}}{\gamma_{m}}}\right)
$$

In (11) together with (5) and (6) form the multi-objective optimization problem (MOOP). There are many methods for solving MOOP including the weighted sum method [25], $\epsilon$-constraint method [26], and multi-objective genetic algorithm method [27]. In particular, the weighted sum method is widely used for its simplicity. In this study, we use the weighted sum method to solve the crowdsourcer's utility maximization problem. We consider the composite function $U$ as a linear combination of the crowdsourcer's utility function for each issue category given by (12),

$$
U=\sum_{c_{j} \in C} w_{c_{j}} u_{c_{j}}
$$

where $w_{c_{j}}$ is a constant, $w_{c_{j}}>0$. The weight $w_{c_{j}}$ is greater for the prioritized issue categories than for those that are not prioritized. We perform function transformation [28] on each crowdsourcer's utility function so that each function has a similar range as (13),

$$
u_{c_{j}}^{t}=\frac{u_{c_{j}}}{\left|u_{c_{j}}^{\prime}\right|}
$$

where $u_{c_{j}}^{t}$ is transformed $u_{c_{j}}$, and $\left|u_{c_{j}}^{\prime}\right|$ is the absolute maximum of $u_{c_{j}}$. The maximum value of $u_{c_{j}}$ exists provided by (14),

$$
\frac{\partial^{2} u_{c_{j}}}{\partial R_{c_{j}}^{2}}=-\frac{\lambda y\left[(1-y) R_{c_{j}}^{y-2} P+R_{c_{j}}^{2 y-2} P^{2}\right]}{\left(1+R_{c_{j}}^{y} P\right)^{2}}<0
$$

for $R_{c_{j}}>0$, where $P=\sum_{i \in S_{j}} T_{i}^{y}$, which is strictly concave. Moreover, $u_{c_{j}}=0$ as $R_{c_{j}}=0$ and $u_{c_{j}} \rightarrow-\infty$ as $R_{c_{j}} \rightarrow \infty$. 
Now, the problem for maximizing (11) with the constraint given by (5) and (6) has turned into the problem of maximizing the transformed composite function

$$
U_{t}=\sum_{c_{j} \in C} w_{c_{j}} u_{c_{j}}^{t}
$$

with those constraints. The following theorem provides the Stackelberg equilibrium on the model of the Stackelberg game.

Theorem 1: Let $w_{c_{j}}>0$ be given, $c_{j} \in C$. There exists the Stackelberg equilibrium $\left(\mathbf{R}^{*}, \mathbf{q}^{n e}\right)$ on the proposed Stackelberg game where $\mathbf{R}^{*}$ maximizes the crowdsourcer utility for each issue category.

Proof 1: For a given category of issue, we have known from the previous discussion that there exists a unique strategy set that maximizes each participant's utility, i.e., the strategy set at Nash equilibrium. Thus, it suffices to show that $\mathbf{R}^{*}$ exists. The constraints (5) and (6) form a compact and convex set on $\mathbb{R}^{M}$, and $U_{t}$ is continuous on the constraints which guarantees the existence of $\mathbf{R}^{*}$. In addition, let us consider the second partial derivatives of $U_{t}$ as (16)

$$
\frac{\partial^{2} U_{t}}{\partial R_{c_{j}}^{2}}=-\frac{w_{c_{j}} \lambda y\left[(1-y) R_{c_{j}}^{y-2} P+R_{c_{j}}^{2 y-2} P^{2}\right]}{\left|u_{c_{j}}^{\prime}\right|\left(1+R_{c_{j}}^{y} P\right)^{2}}<0
$$

and

$$
\frac{\partial^{2} U_{t}}{\partial R_{c_{j}} \partial R_{c_{k}}}=0 \text { for } j \neq k
$$

which form a diagonal Hessian matrix with negative entries so that the Hessian matrix is negative definite. Hence, $U_{t}$ is strictly concave on its domain. Thus, $\mathbf{R}^{*}$ is unique for a given $w_{c_{j}}>0$ and it is on the Pareto front.

\section{SIMULATION RESULTS}

For every number of issue category $M$, we distinguish the issue categories into two categories, i.e., the preference (prioritized) category and nonpreference category. Thus, we might set $M=2$ and the set of issue categories is given by $C=\left\{c_{1}, c_{2}\right\}$ in which $c_{1}$ is preference category and $c_{2}$ is nonpreference category. For each category, the number of participants $N=1000$. We set parameter $\lambda=10$ and $y=0.5$ and perform several simulations by using the CVXPY solver [29] as follows:

- Simulation 1: We let $\gamma_{\min }=0.3$ and $\kappa_{\max }=5$ and assume that the reputation and cost for each participant is randomly uniformly distributed over $\left[\gamma_{\min }, 1\right]$ and $\kappa_{i} \in\left(0, \kappa_{\max }\right]$. We then vary preference parameter $\alpha$ in the range $[0,1]$ with the increment 0.1 . In this case, we solve the trivial optimization problem (not MOOP) by considering an issue category. Figure 3 shows the optimal reward at a certain category increases as the value of $\alpha$ increases. This occurs because increasing $\alpha$ will increase the first term of (11) so that the crowdsourcer utility and the optimal $R$ increase.

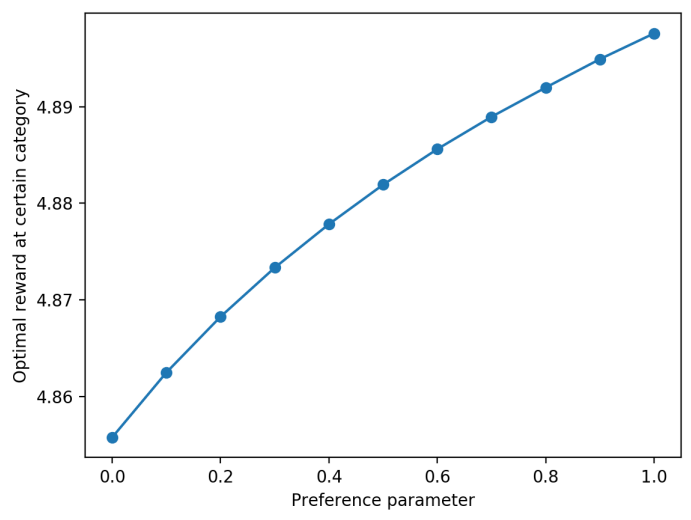

Figure 3. Optimal reward for different preference parameters 
- Simulation 2: We let $\alpha=0.4$. We use similar distribution as in simulation 1 for the reputation and cost of the participant. We plot the pareto front for different $R_{\text {budget }} \in[3,7]$ by an increment of 1 with 1000 data points as shown in Figure 4. Since the budget is limited, the pareto optimal is on $X=\left\{R_{1}, R_{2} \in\right.$ $\left.\mathbb{R}^{2} \mid(1+\alpha) R_{1}+R_{2}=R_{\text {budget }}\right\}$.

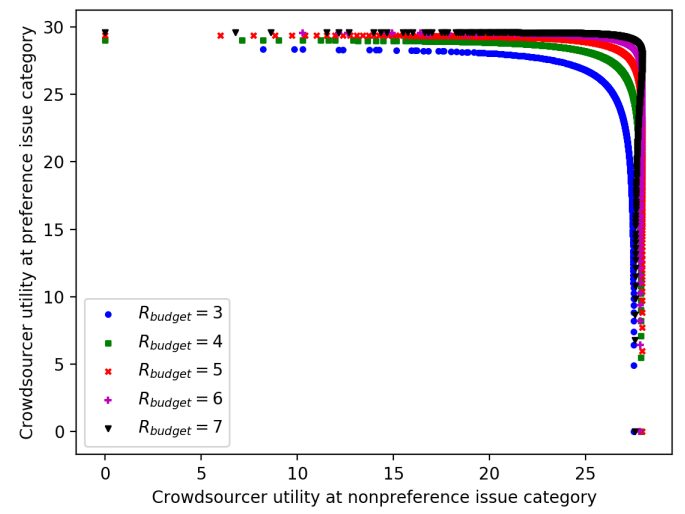

Figure 4. Pareto front for different $R_{\text {budget }}$

- Simulation 3: We let $R_{\text {budget }}=8$ and $\alpha=0.4$. The reputation and cost distribution of the participant is similar to simulation 1 . We vary the weight constant for preference issue category $w_{c_{1}}$ in the range $[1,10]$ by an increment of 1 , and we set the weight constant for the nonpreference issue category $w_{c_{2}}=1$. Thus, the relative weight $\frac{w_{c_{1}}}{w_{c_{2}}}=w_{c_{1}}$. Figure 5(a) represents the graph of the optimal reward of the preference and nonpreference categories of issues for different values of the relative weight. As the relative weight increases, the optimal reward of the preference category increases, but the optimal reward of the nonpreference category decreases as well as the optimal crowdsourcer utility for each category as shown in Figure 5(b). In this case, the budget is more allocated to the prioritized issue as the relative weight increases.

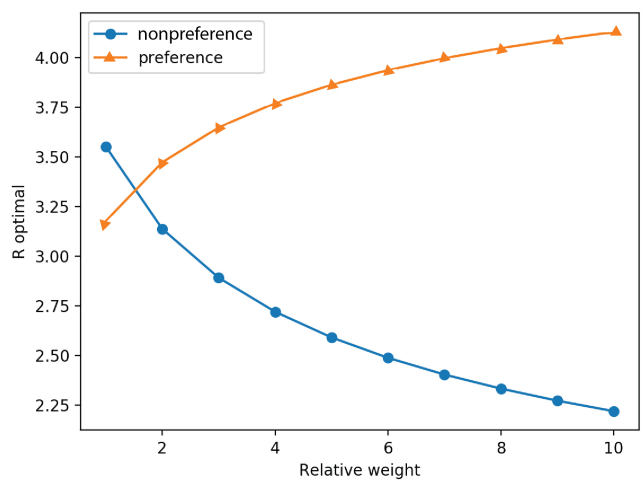

(a)

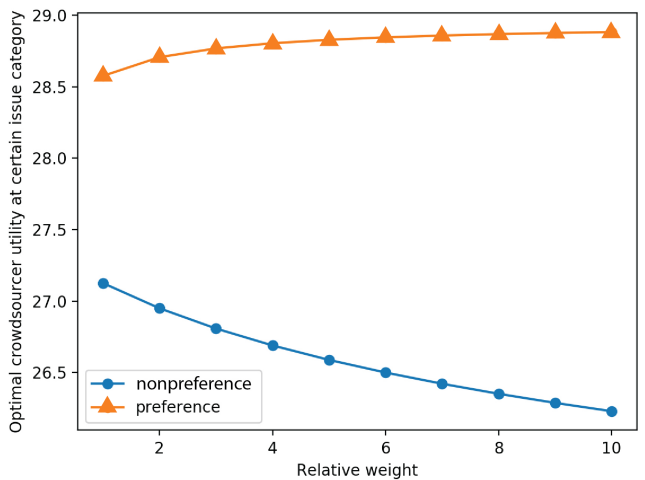

(b)

Figure 5. The optimal value of (a) reward and (b) crowdsourcer utility of preference and nonpreference issue categories for different relative weights

- Simulation 4: We let $R_{\text {budget }}$ be expressed over interval $[1,15]$ with an increment of $1, \alpha=0.4, w_{c_{1}}=5$, and $w_{c_{2}}=1$. The distribution of reputation and cost of each participant is similar to simulation 1 . We examine the sum of the report qualities at Nash equilibrium for each category (preference and nonpreference), the sum of crowdsourcer utilities, and the sum of optimal rewards of those categories over the range of $R_{\text {budget }}$ given by Figure 6(a) to Figure 6(c), respectively. From these figures, we see that after certain $R_{\text {budget }}$ on interval 10 to 12 , the sum of report qualities reported at NE, the sum of crowdsourcer utilities, and the sum of optimal rewards start to increase slowly and are almost constant after $R_{\text {budget }}=12$. The reason is 
that after certain $R_{\text {budget }}^{*}$, the reward for each issue category attains its optimal value such that the optimal reward for $c_{1}$ and the optimal reward for $c_{2}$, denoted by $R_{c_{1}}^{*}$ and $R_{c_{2}}^{*}$, respectively, satisfy the condition $(1+\alpha) R_{c_{1}}^{*}+R_{c_{2}}^{*} \leq R_{\text {budget }}$.

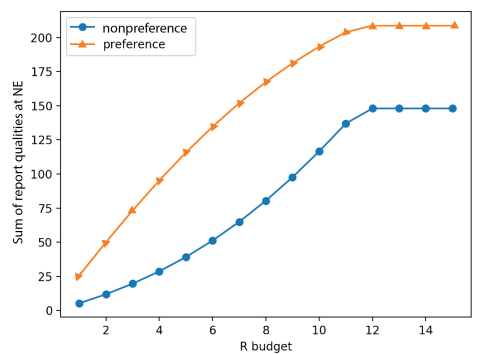

(a)

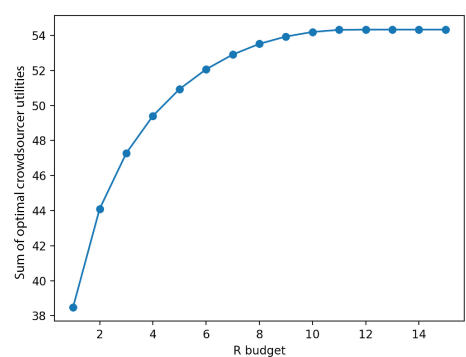

(b)

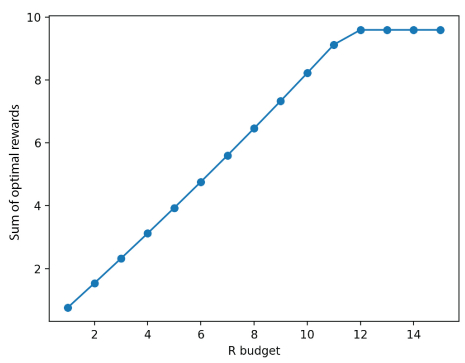

(c)

Figure 6. The sum of (a) report qualities at NE, (b) optimal crowdsourcer utilities, and (c) optimal rewards

$$
R_{\text {budget }}
$$

- Simulation 5: We let $R_{\text {budget }}=10, \alpha=0.2, \gamma_{\min }=0.5, w_{c_{1}}=5$, and $w_{c_{2}}=1$. We vary $\kappa_{\max }$ in the range $[1,10]$ with an increment of 1 and assume that the reputation of each participant is randomly uniformly distributed over $\left[\gamma_{\min }, 1\right]$, whereas its cost is uniformly distributed over $\kappa_{i} \in\left(0, \kappa_{\max }\right]$. Figures 7(a) and 7(b) show the sum of report qualities at NE for each category and the sum of crowdsourcer utilities for different $\kappa_{\max }$, respectively, where these sums decrease as $\kappa_{\max }$ increases as mentioned in [7].

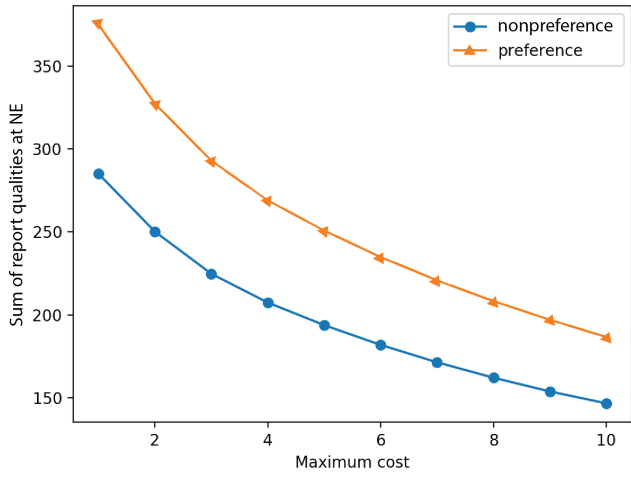

(a)

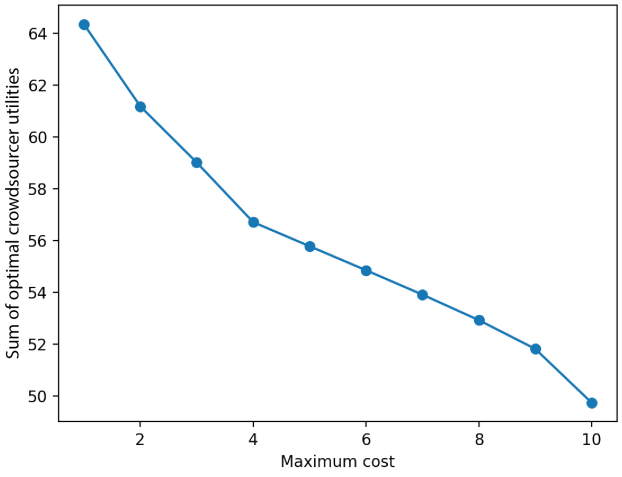

(b)

Figure 7. The sum of (a) report qualities at NE and (b) crowdsourcer utilities for different $\kappa_{\max }$

\section{CONCLUSION}

In this study, we designed an incentive mechanism for mobile crowdsensing based on several identified incentive parameters using the Stackelberg game model. We designed the incentive mechanism that classified incentives according to the issue categories and motivated participants to report the prioritized issue. In addition, it considered the participant's reputation. We used multi-objective optimization to provide the maximization of the crowdsourcer utility in each category of the issue which corresponds to the regional working unit for a given allocated budget. The simulation indicated that the result appropriately corresponds to the theoretical properties of the model.

\section{ACKNOWLEDGEMENT}

This work was supported in part by Indonesian Ministry of Research, Technology, and Higher Education under WCU Program managed by Institut Teknologi Bandung. We thank Pujo Laksono for his helpful 
advice on the AHP method and grammar checking on the manuscript. We thank Henokh Lugo Hariyanto for valuable discussion and advice on the incentive model, also for technical help in preparation for writing the manuscript. We thank Hadi Muhshi for useful discussion and technical help during the simulation.

\section{REFERENCES}

[1] I. M. A. Sanjaya, S. H. Supangkat, and J. Sembiring, "Citizen reporting through mobile crowdsensing: A smart city case of B," in 2018 International Conference on ICT for Smart Society (ICISS), oct 2018, pp. 1-4, doi: 10.1109/ictss.2018.8549976.

[2] M. H. Cheung, F. Hou, and J. Huang, "Participation and reporting in participatory sensing," in 2014 12th International Symposium on Modeling and Optimization in Mobile, Ad Hoc, and Wireless Networks (WiOpt), 2014, pp. 357-364, doi: 10.1109/wiopt.2014.6850320.

[3] Q. Li and G. Cao, "Providing privacy-aware incentives in mobile sensing systems," IEEE Transactions on Mobile Computing, vol. 15, no. 6, pp. 1485-1498, Jun 2016, doi: 10.1109/tmc.2015.2465375.

[4] T. Luo, S. S. Kanhere, and H.-P. Tan, "SEW-ing a simple endorsement web to incentivize trustworthy participatory sensing," in 2014 Eleventh Annual IEEE International Conference on Sensing, Communication, and Networking (SECON), 2014, pp. 636-644, doi: 10.1109/sahen.2014.6990404.

[5] F. Restuccia, S. K. Das, and J. Payton, "Incentive mechanisms for participatory sensing," ACM Transactions on Sensor Networks, vol. 12, no. 2, pp. 1-40, May 2016, doi: 10.1145/2888398.

[6] K. Deb and K. Deb, "Multi-objective optimization," in Search Methodologies, Springer US, Jul 2013, pp. 403-449, doi: 10.1007/978-1-4614-6940-7_15.

[7] D. Yang, G. Xue, X. Fang, and J. Tang, "Incentive mechanisms for crowdsensing: Crowdsourcing with smartphones," IEEE/ACM Transactions on Networking, vol. 24, no. 3, pp. 1732-1744, Jun 2016, doi: 10.1109/tnet.2015.2421897.

[8] J. Y. Koh, G. W. Peters, I. Nevat, and D. Leong, "Privacy considerations in participatory data collection via spatial stackelberg incentive mechanisms," Methodology and Computing in Applied Probability, vol. 23, no. 3, pp. 10971128, Jul 2020, doi: 10.1007/s11009-020-09798-7,

[9] S. Luo, Y. Sun, Y. Ji, and D. Zhao, "Stackelberg game based incentive mechanisms for multiple collaborative tasks in mobile crowdsourcing," Mobile Networks and Applications, vol. 21, no. 3, pp. 506-522, Dec 2015, doi: 10.1007/s11036-015-0659-3.

[10] X. Kang and Y. Wu, "Incentive mechanism design for heterogeneous peer-to-peer networks: A stackelberg game approach," IEEE Transactions on Mobile Computing, vol. 14, no. 5, pp. 1018-1030, May 2015, doi: 10.1109/tmc.2014.2343628.

[11] W. Wu, W. Wang, M. Li, J. Wang, X. Fang, Y. Jiang, and J. Luo, "Incentive mechanism design to meet task criteria in crowdsourcing: How to determine your budget," IEEE Journal on Selected Areas in Communications, vol. 35, no. 2, pp. 502-516, Feb 2017, doi: 10.1109/jsac.2017.2659278.

[12] Y. Zhan, C. H. Liu, Y. Zhao, J. Zhang, and J. Tang, "Free market of multi-leader multi-follower mobile crowdsensing: An incentive mechanism design by deep reinforcement learning," IEEE Transactions on Mobile Computing, vol. 19, no. 10, pp. 2316-2329, Oct 2020, doi: 10.1109/tmc.2019.2927314.

[13] J. Nie, J. Luo, Z. Xiong, D. Niyato, and P. Wang, "A stackelberg game approach toward socially-aware incentive mechanisms for mobile crowdsensing," IEEE Transactions on Wireless Communications, vol. 18, no. 1, pp. 724-738, Jan 2019, doi: 10.1109/twc.2018.2885747.

[14] X. Zhang, Z. Yang, W. Sun, Y. Liu, S. Tang, K. Xing, and X. Mao, "Incentives for mobile crowd sensing: A survey," IEEE Communications Surveys \& Tutorials, vol. 18, no. 1, pp. 54-67, 2016, doi: 10.1109/comst.2015.2415528.

[15] G. Gao, J. Wu, M. Xiao, and G. Chen, "Combinatorial multi-armed bandit based unknown worker recruitment in heterogeneous crowdsensing," in IEEE INFOCOM 2020 - IEEE Conference on Computer Communications, 2020, pp. 179-188, doi: 10.1109/INFOCOM41043.2020.9155518.

[16] Y. Wu, F. Li, L. Ma, Y. Xie, T. Li, and Y. Wang, "A context-aware multiarmed bandit incentive mechanism for mobile crowd sensing systems," IEEE Internet of Things Journal, vol. 6, no. 5, pp. 7648-7658, Oct 2019, doi: 10.1109/jiot.2019.2903197.

[17] H. Jin, L. Su, B. Ding, K. Nahrstedt, and N. Borisov, "Enabling privacy-preserving incentives for mobile crowd sensing systems," in 2016 IEEE 36th International Conference on Distributed Computing Systems (ICDCS), 2016, pp. 344-353, doi: 10.1109/ICDCS.2016.50.

[18] Y. Liu, H. Li, G. Zhao, and J. Duan, "Reverse auction based incentive mechanism for location-aware sensing in mobile crowd sensing," in 2018 IEEE International Conference on Communications (ICC), 2018, pp. 1-6 doi: 10.1109/ICC.2018.8423009.

[19] J. Y. Koh, G. W. Peters, D. Leong, I. Nevat, and W.-C. Wong, "Privacy-aware incentive mechanism for mobile crowd sensing," in 2017 IEEE International Conference on Communications (ICC), 2017, pp. 1-6, doi: 10.1109/icc.2017.7997418.

[20] A. Chakeri and L. G. Jaimes, "An incentive mechanism for crowdsensing markets with multiple crowdsourcers," IEEE 
Internet of Things Journal, vol. 5, no. 2, pp. 708-715, Apr 2018, doi: 10.1109/jiot.2017.2706946.

[21] L. Wang, Z. Yu, Q. Han, B. Guo, and H. Xiong, "Multi-objective optimization based allocation of heterogeneous spatial crowdsourcing tasks," IEEE Transactions on Mobile Computing, vol. 17, no. 7, pp. 1637-1650, Jul 2018, doi: 10.1109/tmc.2017.2771259.

[22] Y. Zhang, X. Zhang, and F. Li, "BiCrowd: Online biobjective incentive mechanism for mobile crowdsensing," IEEE Internet of Things Journal, vol. 7, no. 11, pp. 11078-11091, Nov 2020, doi: 10.1109/jiot.2020.2994365.

[23] L. M. Bettencourt, "The uses of big data in cities," Big Data, vol. 2, no. 1, pp. 12-22, Mar 2014, doi: 10.1089/big.2013.0042.

[24] A. Bar-Noy, G. Cirincione, R. Govindan, S. Krishnamurthy, T. F. LaPorta, P. Mohapatra, M. Neely, and A. Yener, "Quality-of-information aware networking for tactical military networks," in 2011 IEEE International Conference on Pervasive Computing and Communications Workshops (PERCOM Workshops), pp. 2-7, doi: 10.1109/percomw.2011.5766887.

[25] R. T. Marler and J. S. Arora, "The weighted sum method for multi-objective optimization: new insights," Structural and Multidisciplinary Optimization, vol. 41, no. 6, pp. 853-862, Dec 2009, doi: 10.1007/s00158-009-0460-7.

[26] M. Parvizi, E. Shadkam, and N. Jahani, "A hybrid coa $\epsilon$-constraint method for solving multi-objective problems," International Journal in Foundations of Computer Science \& Technology, vol. 5, no. 5, pp. 27-40, Sep 2015, doi: 10.5121/ijfcst.2015.5503.

[27] K. Deb, A. Pratap, S. Agarwal, and T. Meyarivan, "A fast and elitist multiobjective genetic algorithm: NSGA-II," IEEE Transactions on Evolutionary Computation, vol. 6, no. 2, pp. 182-197, Apr 2002, doi: 10.1109/4235.996017.

[28] R. Marler and J. Arora, "Survey of multi-objective optimization methods for engineering," Structural and Multidisciplinary Optimization, vol. 26, no. 6, pp. 369-395, Apr 2004, doi: 10.1007/s00158-003-0368-6.

[29] S. Diamond and S. Boyd, "Cvxpy: A python-embedded modeling language for convex optimization," The Journal of Machine Learning Research, vol. 17, no. 1, pp. 2909-2913, 2016. 